\title{
La traduction des classiques comme historiographie : herméneutique, temporalité, "poièsis
}

\section{LUDOVICA MAGGI}

DOI : https://doi.org/10.31577/WLS.2021.13.3.3

\section{TRADUIRE LES CLASSIQUES : HERMÉNEUTIQUE DE L'CEUVRE DANS LE TEMPS}

Le point de départ de notre réflexion sur la traduction des classiques est l'herméneutique de l'œuvre. En d'autres termes : nous posons que la traduction d'un classique est la manifestation de la compréhension interprétative de ce dernier par le traducteur.

Dans le champ disciplinaire des études sur la traduction, l'herméneutique n'a pas manqué d'être invoquée comme cadre de référence. $S$ 'inscrivent notamment dans ce filon, outre la pensée de la motion herméneutique (Steiner 1975), la théorie interprétative de la traduction (Seleskovitch et Lederer 1984, en premier lieu) et l'herméneutique traductive allemande (Stolze 1992, entre autres). Ces constructions théoriques font du texte le terrain de jeu de l'herméneutique et tracent les limites de cette dernière à l'endroit même où ils relèvent sa spécificité - c'est à dire, dans le sujet. En effet, tout en mettant l'accent sur l'action de compréhension réalisée par le sujet traduisant et tout en reconnaissant la nécessaire contribution de ses compétences et de ses connaissances à l'advenir de la compréhension, elles reposent sur l'idée que l'objet même de la compréhension est un sens fondamentalement intersubjectif, avec lequel le sujet traduisant ne saurait - ni, en aucun cas, ne devrait - interférer.

L'herméneutique traductive que nous mettons au centre de notre pensée de la traduction des classiques dépasse ce périmètre en ceci qu' elle a affaire à l'œuvre au-delà du texte et qu'elle assume la pleine subjectivité du sujet traduisant. Dans cette optique,

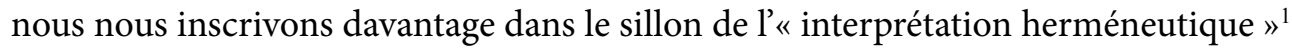
de Jean-René Ladmiral $(2006,112)$ et de l'« inscription interprétative » ${ }^{2}$ de Lawrence Venuti $(2013,18)$.

Reposant sur le sujet, notre herméneutique de l'œuvre ne le relègue pas pour autant dans son individualité. Au contraire, elle l'inscrit dans un tissu culturel commun qui ne manque pas de contribuer à l'exercice de son action interprétative. S'agissant spécifiquement de la traduction des classiques, nous accueillons dans l'herméneutique subjective de l'œuvre l'intuition clé de l'esthétique collective de la réception : l'idée que la compréhension de l'œuvre du passé est également le fruit d'une posture herméneutique partagée, historiquement située et, de ce fait, nécessairement évolutive (Jauss 2015, 43). 
C'est sur ces bases que nous allons à la rencontre de l'herméneutique philosophique de Hans-Georg Gadamer (1996, 1999a et 1999b). De cette "phénoménologie de l'évènement de la compréhension " (Grondin 2004, 15) nous retenons les concepts fondateurs de cercle herméneutique et de fusion des horizons. Dans l'optique d'une herméneutique subjective de l'œuvre, ces schémas nous livrent l'idée essentielle que toute compréhension se fait à partir d'un point de départ propre au sujet comprenant : son préjugé d'une part - ce qu'il connaît déjà et qui l'aide à faire la lumière sur la chose à comprendre ; son horizon d'autre part - membrane intellective de contact entre le sujet connaissant et l'objet dont il fait la connaissance.

Dans la perspective spécifique de la traduction des classiques il convient de mettre en exergue, en résonnance avec la leçon de Jauss, le lien de ces interfaces avec le temps du traducteur. Sur ce point, nous retenons les considérations de deux commentateurs du philosophe allemand. Deniau précise notamment qu'au sein du système gadamérien, le préjugé est "lié à notre situation historique " : puisqu' " appartenir à une histoire, à une tradition, c'est être situé, avoir un point de vue »(Deniau 2002, 339), "le préjugé, en tant que structure médiatrice, désigne [...] l'historicité de la compréhension » (336). Michael Pickering rappelle quant à lui, dans un article au titre significatif de « History as Horizon: Gadamer, Tradition and Critique », à quel point pour Gadamer la vérité est historiquement conditionnée et en cela provisoire : tout acte de recherche est marqué par l'historicité de celui qui l'accomplit, par la temporalité de son être dans le monde. Plus précisément, le présent du sujet interprétant intervient dans la constitution du préjugé qui constitue l'interface de médiation pour toute connaissance. Le passé du sujet est aussi mobilisé à cet effet $(1999,180)$. Le temps se définissant ainsi comme horizon herméneutique, Gadamer nous offre les clés pour faire de la traduction des classiques un acte d'herméneutique dans le temps, qui se nourrit du regard présent porté sur l'œuvre par le traducteur tout en recevant le legs interprétatif du passé.

La dépendance entre l'acte traductif et le temps de sa réalisation n'est pas, en tant que telle, une acquisition nouvelle en traductologie. S'inscrivant dans la mouvance gadamérienne et se prononçant, entre autres, sur la question de la traduction dans le temps, Jane Elisabeth Wilhelm $(2006,4)$ la relie notamment à la théorie des normes (Toury 1980) et rappelle que les pratiques traductives évoluent au fil du temps en fonction des conventions de lecture et d'écriture. Charles Le Blanc s'érige également en porte-parole de l'action interprétative de la traduction dans le temps et de la dépendance de cette dernière des normes scripturales propres aux différentes époques. En ce sens, il précise d'une part que l'action fondatrice de la traduction est la lecture de l'œuvre et que celle-ci se renouvelle constamment dans le temps $(2019,2013)$; d'autre part, il signale que «le besoin de retraduire les œuvres correspond à l'évolution naturelle de la langue [...], de ses métamorphoses rhétoriques » $(2013,265)$.

\section{TRADUIRE LES CLASSIQUES : HERMÉNEUTIQUE DU TEMPS}

Notre cadre théorique tente d'apporter un complément à ces bases. En effet, parallèlement à la réflexion sur la traduction comme herméneutique dans le temps, il y a lieu, nous semble-t-il, de poser la question spécifique de la traduction des 
classiques en termes d'herméneutique $d u$ temps. Autrement dit, de définir le temps comme l'objet même de la démarche herméneutique du sujet traduisant. Traduire les classiques serait ainsi, en premier lieu, s'interroger sur la nature de l'œuvre en tant qu'objet du passé, ce qui à son tour impliquerait de prendre position sur le passé et sur sa relation au présent.

Un tel développement invite la réflexion sur la traduction des classiques à se rapprocher des théories de l'histoire. Plus spécifiquement, nous ouvrons notre cadre théorique aux régimes de temporalité et osons affirmer leur contribution à la définition de l'horizon herméneutique du traducteur. Nicolas Offenstadt $(2011,14)$ présente ces catégories de la pensée comme « les modes de rapport au temps, les expériences du temps "; " la manière avec laquelle les cultures structurent leur représentation du temps " et, plus précisément, comme "la façon dont les contemporains articulent les trois temps, passé présent futur ». François Hartog $(2015,13)$ lui fait écho, précisant la relation réciproque des plans temporaux au sein de ces représentations du temps et suggérant que celle-ci peut se manifester sous forme de concurrence : à ses yeux, « un régime d'historicité n'est ainsi qu'une façon d'engrener passé, présent et futur ou de composer un mixte des trois catégories [...] un des trois composants étant de fait dominant ». Hartog signale aussi l'évolution de l'expérience du temps au fil des siècles - d'un référentiel préhistorique à caractère cyclique, à la fois sans temps et toujours présent ; à la conception ancienne du passé comme source de vérité et d'enseignement ; à l'orientation moderne vers le futur et le progrès ; jusqu'à l'extinction de la foi dans l'avenir au cœur d'une société contemporaine "présentiste " centrée sur l'éphémère et le court-terme.

La réflexion sur le rapport aux temps mérite d'être complétée par la pensée du rapport à l'histoire, en tant qu'enquête sur le passé. De ce point de vue, Jacques Le Goff (1988) nous apprend à entrevoir la présence de ce qui pourrait aisément être identifié comme un horizon herméneutique pour tout regard jeté sur le passé : une grille de lecture qui guide l'historien dans son action de recherche et de mise en relation des informations ; un sens donné à l'histoire qui se déroule et qui s'écrit - de l'historicisme positiviste, qui prive l'histoire de toute fonction éthique pour en faire une science exacte, documentaire, érudite, à la nouvelle histoire, qui remplace « l'histoire-récit par l'histoire-problème » (329-330) s'ouvrant aux méthodes d'enquête des sciences sociales.

L'apport de ces contributions à notre réflexion sur la traduction des classiques comme exercice herméneutique sur le passé est double. D’une part, elles corroborent la thèse que le temps - et plus spécifiquement le temps présent - en tant qu'horizon de départ, est un facteur crucial pour la compréhension du passé. Comme le formule explicitement Le Goff : « toute histoire est bien contemporaine dans la mesure où le passé est saisi dans le présent et répond donc à ses intérêts » (223) ; "l'historien part de son présent pour poser des questions au passé » (190). Le présentisme d'Hartog renforce, quant à lui, en référence à l'époque contemporaine, le constat de la prépondérance du point de vue présent dans l'herméneutique du passé. Sur le plan traductologique, cela implique d'assumer que toute traduction s'accompagne nécessairement d’une projection du présent sur le passé et, partant, d’une attraction 
du passé vers le présent. D’autre part, les considérations de ces auteurs nous poussent à interroger qualitativement les démarches herméneutiques des traducteurs des classiques pour saisir les spécificités des régimes de temporalité qui les soutiennent, au-delà de l'empreinte indéniable du présent. Si les postures vis-à-vis du passé sont potentiellement illimitées, il nous semble toutefois raisonnable d'envisager un certain nombre d'attitudes récurrentes.

Dans cette optique, il convient d'évoquer les balises posées par Alexis Nouss (2007, 2008, 2009). À partir d'une intuition qui rejoint la nôtre - les pratiques traductives reflètent, entre autres, les postures chronologiques d'une société donnée (2009) -, ce traductologue procède à une catégorisation binaire des manières traductives en métahistorique et transhistorique, « la première cherchant à édifier un ordre de réalité au-dessus du flux temporel et la seconde visant à établir en son sein une relation entre différentes historicités » (297). Plus particulièrement, dans son schéma conceptuel, « le dialogue (transhistorique) entre des historicités chronologiquement distinctes et distantes » $(2008 \mathrm{~b}, 385)$, « superpose les strates chronologiques », " reprend du passé ce qui lui semble encore actuel » (386), au nom de "l'imprégnation d'une époque par une époque antérieure, au sens premier de fécondation » (384). L'approche métahistorique, en revanche, neutraliserait l'altérité temporelle pour signifier l'universalisme.

Si ces propos suggèrent une vision innovante du traitement de la temporalité en traduction, le développement de ces prémisses ne problématise pas réellement l'expérience du passé et demeure plutôt dans le périmètre connu de l'antinomie formelle sourcisme - ciblisme. Ainsi, affirme-t-il, « le transhistoricisme [...] est étayé par l'attention à la forme tandis que le métahistoricisme la néglige » (Nouss 2009, 298). Plus précisément, Nouss rapproche le transhistoricisme de «l'hyperlittéralisme traductionnel » (298) à la Johann Wolfgang Goethe ou à la Walter Benjamin (2008a) et le métahistoricisme - non sans amalgames - aux belles infidèles de Nicolas Perrot D'Ablancourt (2008b, 385) comme à la «traduction sémantique ou communicative qui se préoccupe du sens en négligeant la forme » $(2009,298)$.

Et pourtant, lidée que la traduction puisse véhiculer une approche au passé, voire une philosophie de l'histoire, a un intérêt certain pour l'étude de la gestion de la temporalité en traduction. De ce fait, elle mérite à nos yeux d'être reliée au fil théorique de l'herméneutique pour poser les bases d'un discours sur la traduction des classiques qui dépasse l'alternative formelle archaïsation-modernisation (Steiner 1975, 334-356; Holmes 1994 ; Lefere 1994 ; Crisafulli 1999 ; Piletić 1999 ; Rega 2001 ; Roux-Faucard 2001) et contourne l'association de ce couple d'opposés avec l'antithèse culturelle étrangéisation-domestication (Schleiermacher [1813] 1963 ; Venuti 1998 ; Paloposki 2011) et son corollaire idéologique respect-négligence (Berman 1984, 1999 ; Venuti 1995, 1998 ; Aaltonen 1993 notamment ; Laera 2014, 2016). Autrement dit, sur la base de cette idée première, il devrait être possible d'abandonner la logique selon laquelle une traduction archaïsante serait respectueuse de la passéité formelle, et partant, de la passéité tout court de l'œuvre traduite, alors qu'un choix modernisant témoignerait d'une volonté de la profaner au profit d'un rapprochement de facilité de l'œuvre source avec les référentiels formels et conceptuels du public cible. 
Offrant une issue de cet aut aut, l'herméneutique du temps devrait pouvoir mener en premier lieu à une prise de conscience : toute traduction d'une œuvre d'hier réalisée aujourd'hui s'inscrit, par définition, dans une démarche de "modernisation ", en raison du fait qu'elle émane d'un horizon herméneutique présent. Deuxièmement, elle devrait pouvoir nous inviter à lire les choix traductifs, et donc la forme de la traduction, comme des indicateurs d'une relation au passé - subjective et historiquement située - qui ne saurait se réduire à l'alternative respect-négligence du statut de l'œuvre en tant qu'objet du passé.

Prenant en partie appui sur la terminologie de Nouss et tout en redéfinissant ses champs sémantiques dans l'optique herméneutique que nous venons d'esquisser, nous proposons d'envisager au moins trois grandes familles d'approches au temps dans la traduction des classiques. La première serait historiciste, visant à rendre compte du classique en tant qu'objet appartenant au passé et à documenter les traits spécifiques de sa passéité, tels que le traducteur la comprend. La deuxième approche serait métahistorique, visant à désolidariser le classique de son époque propre pour affirmer sa capacité à signifier à toute époque, y compris dans le présent. La troisième approche serait transhistorique, en ceci qu'elle s'inscrirait dans une démarche d'interaction, voire d'interpolation, assumée et affichée entre le présent et le passé.

Ces trois grandes tendances herméneutiques semblent trouver résonnance dans la littérature critique concernant les classiques, qui permet d'identifier plusieurs manières de penser le classique : tel un objet inactif, qui serait à ignorer (classique «oblitéré ", dirions-nous - Settis 2004, 8-9 ; Dionigi 2002, 7 ; Mieli 2002, 171), à étudier et conserver comme témoin de son temps (classique « document "-Vegetti 2006, 270-271) ou à respecter et célébrer en tant qu'œuvre remarquable du passé (classique « excellent »-Sainte-Beuve 1850 ; Eliot 1944) ; tel un objet actif : à commémorer pour sa contribution à l'héritage culturel commun (classique " patrimoine »-Dionigi 2002, 9 ; Santagata 2002, 268), à lire et relire pour en tirer un plaisir esthétique et/ou y retrouver des valeurs qui ne sauraient s'épuiser au fil du temps (classique "permanent " - Calvino 1991, 7-12) ; tel un objet interactif, interprétable à volonté et ouvert au palimpseste des poétiques en traduction (classique « réceptionnel »- Kermode 1975 ; Lianeri et Zajko 2008, 4 ; Martindale 1993, 2013 ; Goldhill 2010).

Dans cette construction interprétative et polycentrique - qui pourrait logiquement conduire à envisager la traduction du classique dans son ensemble comme une expérience transhistorique, de traversée et de contamination des temps - la grille de lecture formelle et bipolaire archaïsation-modernisation ne peut être appliquée avec aisance. En effet, chaque regard posé sur le passé suggère une écriture traductive singulière, à étudier à travers un large spectre afin de saisir les modes de sa modernisation incontournable, de préférence en association avec le paratexte, à la recherche d'une éventuelle argumentation de ces choix.

Nous avons mené cet exercice sur quelques traductions italiennes contemporaines de Phèdre de Racine (Maggi 2019). Nous avons notamment découvert une volonté documentaire derrière la traduction limpide du metteur en scène Luigi Nazareno Todarello (Racine 2011), qui a souhaité se doter d'une base linguistique neutre et pleinement accessible à l'oreille contemporaine pour mettre davantage en relief, 
dans le cadre de la performance, la lecture rythmique du texte, selon un procédé reconstitutif de la déclamation théâtrale baroque (Todarello 2011). La traduction de Roberto Carifi (Racine 1993), épurée, en rime, dans un registre linguistique quelque peu formel, nous a semblé vouloir en même temps évoquer, dans un élan historiciste, l'équilibre imprenable du classicisme tel que Racine le fixe en France au XVII siècle, et capturer l'essence intemporelle de l'ouvrage traduit. Giuseppe Ungaretti (1950) et Giovanni Raboni (Racine 1984) nous ont, quant à eux, révélé un travail transhistorique remarquable. Ungaretti évoque, tout en le fractionnant, le rythme syntaxique et métrique de l'alexandrin, tandis qu'il met en place, sur le plan du lexique, une coexistence entre le contemporain concret et le classique évanescent. Il impose ainsi au texte, par la traduction, la marque d'une brisure existentielle et temporelle, faisant de Phèdre une ouvre de la douleur intime de fin de vie - qui n'est pas sans évoquer le désarroi avant-gardiste du plus jeune âge du poète - et de la décadence baroque, sorte d'objet poétique survivant aux ruines littéraires du classicisme. Raboni déséquilibre le rythme et suspend la langue, se servant du dispositif de l'étrangement pour signifier paradoxalement, par l'hyper-contemporain, la distance du classique par rapport au temps culturel de sa réception, tout en définissant, d'un regard historiciste, la retenue - le non-dit propre de la société racinienne - en tant que trait saillant de la pièce. ${ }^{3}$

\section{TRADUIRE LES CLASSIQUES : HISTORIOGRAPHIE (OU « HISTORIOPOÉSIE " ?)}

Les deux premiers mouvements de notre réflexion nous ont permis d'envisager la traduction des classiques comme un acte herméneutique du temps inscrit dans le temps. Un troisième volet s'impose, pour combler l'écart entre interprétation et écriture, pour contempler la mise en mot de ce qui a été compris.

En ce sens, un rapprochement peut être fait entre la traduction des classiques comme écriture interprétative des œuvres du passé et l'historiographie, au sens d'écriture de et sur l'histoire. Paul Ricœur (2000) nous rappelle à cet effet que l'historiographie est un processus en trois temps, articulé en une phase documentaire, une phase explicative/compréhensive, une phase scripturale/représentative. La première consiste en la collecte des documents à étudier, «l'établissement de la preuve documentaire " $(2000,169)$; la deuxième porte sur la compréhension des faits et des dynamiques du passé et vise à définir le pourquoi du passé ; la troisième correspond à l'écriture historique proprement dite et peut se définir comme " phase littéraire ou scripturale lorsqu'il s'agit du mode d'expression, phase représentative lorsqu'il s'agit de l'exposition, de la monstration, de l'exhibition de l'intention historienne prise dans l'unité de ses phases, à savoir la représentation présente des choses absentes du passé » (171). Ainsi, l'écriture historique peut être entendue « aussi bien comme mise en intrigue, disposition narrative qu'explication à proprement parler » (236). Au sujet de cette troisième phase, Ricœur précise la double connotation portée par le verbe représenter : présenter, illustrer (au sens de vorstellen), mais aussi tenir lieu de (au sens de vertreten). Dans cette deuxième acception, l'écriture historiographique acquiert une fonction de « représentance », « lieutenance » du passé (Ricœur 1985, 253-254). 
Le parallélisme avec la traduction des classiques nous semble évident : en tant que travail concernant un document du passé, elle se fonde, comme l'historiographie, sur l'établissement de la source documentaire (le texte source, mais également tous les paratextes nécessaires à sa compréhension en tant que texte et en tant qu'œuvre); puis elle comporte un temps herméneutique de compréhension ; enfin elle impose une écriture.

$\mathrm{Au}$-delà de la ressemblance entre traduction et historiographie en termes de processus, c'est l'écriture traductive qui mérite à nos yeux d'être placée au centre de l'attention comme facteur clé de l'action historiographique de la traduction. Véhicule de l'expérience herméneutique du traducteur, celle-ci façonne en effet l'identité de l'œuvre en tant qu'objet du passé dans le présent de la réception.

Dans notre cadre théorique, l'écriture traductive est poiétique. Dérivé de poièsis - mot aristotélicien, substantif du verbe poiein, « faire », «façonner » (Martin 2020) -, ce terme a pour nous une portée multiple. Parce qu'elle affiche l'action créative du traducteur, appelé à se confronter avec un produit artistique et à le reconstruire en tant que tel pour le public de destination, la notion de poïétique le définit premièrement en tant qu'auteur du classique traduit (Berman 1995; Bassnett et Bush 2006 viennent ici évidemment à l'esprit). Elle reconnait également au traducteur une poétique propre. En ce sens, elle implique la présence, chez le traducteur - en synergie, voire en symbiose, avec son horizon herméneutique - d'un parti pris traductologique en termes d'éthique traductive - quelle fonction pour la traduction, quel rôle et quelle place pour le traducteur d'un classique ? - et de projet de traduction - quelle écriture pour quel public dans quel contexte ? (Berman 1995 plus que Lefevere 1992, chargé d'une nuance idéologique que nous ne souhaitons pas revendiquer.)

Or, dans l'écriture traductive la poièsis est aussi et surtout gestion de la parole, au sens que Henri Meschonnic (1982a) - sans référence au cadre herméneutique a voulu attribuer à l'objet premier de la traduction : la forme dans son agencement, dans son mouvement, dans sa vocation à la vocalisation. De cette poétique - qui relève du rythme ou, en synonymie, de l'oralité (Meschonnic 1982b) et qui n'est pas sans rappeler la rhétorique de Le Blanc, cette « façon de rythmer, cadencer, diviser, mettre en scène le texte pour la lecture " (Le Blanc 2013,265) - nous retenons tout particulièrement la référence au champ de l'oralité. Par ce biais, nous élargissons l'espace sémantique du rythme aux choix linguistiques, au potentiel de mise en voix, ainsi qu'au discours porté par la parole traductive (Maggi 2019). Nous mettons la poétique du rythme au centre de notre réflexion traductologique, en attribuant à la gestion de la parole en traduction la fonction de pierre de touche de l'herméneutique du traducteur. Et si Meschonnic nous apprend à prendre en compte cette dimension, nous le faisons non pas avec l'œil « rythmiquement sourcier » de celui qui demande à la traduction de documenter le rythme du classique, mais avec un regard cibliste qui demande au rythme de la traduction de révéler l'herméneutique du temps et du classique propre au traducteur.

Enfin, l'écriture traductive est poḯtique car elle «fait » le classique, elle le pétrit en tant qu'objet du passé, elle le place, pour le public cible, dans l'une ou l'autre des catégories de l'herméneutique du temps que nous avons tenté d'explorer plus haut. 
En ce sens, nous nous souvenons du message de Michel de Certeau, qui fait de l'écriture de l'histoire (1975) une pratique historique présente et conquérante, visant moins à rendre compte du passé qu'à le construire.

Poḯtique, l'écriture de la traduction représente donc son objet comme l'écriture de l'histoire à la manière dont l'entend Ricœur : elle produit un classique qui s'érige en lieu et place de l'œuvre source; et en même temps elle la relate, ou plutôt elle la raconte, en produisant un discours sur elle et sur son temps. Ainsi, elle construit pour les contemporains une image de cette œuvre. En ce sens, il nous semble pertinent de rechercher, dans l'étymologie du terme « histoire », la racine *id du verbe " voir », qui fait de l'historiographie le récit de ce qui a été vu. Et de nous souvenir, avec Canfora (2000), qu'aux origines de la discipline se trouve justement le témoignage visuel, apprécié plus que le ouï-dire, en tant que source d'informations et gage de fiabilité.

Sur ces bases, il nous serait possible de conclure que la traduction des classiques est bel et bien une écriture de l'histoire, une historiographie, donc. En osant davantage, nous pourrions qualifier l'acte neutre du graphein en le chargeant de la force poïétique de l'écriture traductive, pour faire de l'écriture traductive des classiques une « historiopoésie».

\section{CONCLUSION}

Avant de conclure notre réflexion, nous nous octroyons un corollaire, qui ouvre le récit de l'histoire de l'œuvre - déjà désobjectivé par le biais de l'action herméneutique et poiétique de la traduction - à l'univers ouvertement subjectif de la mémoire.

En effet, si dans son travail de construction de l'image du classique, la démarche historiographique de la traduction s'appuie principalement sur les sources - en l'occurrence, le texte de départ - et sur leur interprétation, elle rencontre aussi une autre dimension, à la fois complémentaire et concurrente : la mémoire du passé. Individuelle et/ou collective, celle-ci repose non pas sur le document, mais sur l'image (sic!) retenue d'un passé dont on aurait fait l'expérience ou reçu le témoignage, ou bien que l'on aurait appris grâce au récit historiographique. Devenue au $\mathrm{XX}^{\mathrm{e}}$ siècle un objet d'étude à part entière de l'historiographie (Le Goff 1988) en tant que phénomène anthropologique et social présidant à la réception des faits historiques, à leur élaboration, à la définition des identités et jusqu'à l'expérience du présent et à la vision de l'avenir (Monteforte 2019), la mémoire mérite d'être intégrée dans notre lecture historiographique de la traduction, en raison de son pouvoir de réfraction de l'image du passé.

La mémoire peut ainsi entrer en jeu dans la traduction des classiques à double titre : comme préjugé potentiel de l'interprétation du classique, capable d'orienter la démarche herméneutique, et comme destination possible de la traduction. En effet, dès lors que l'on reconnait à cette dernière, en vertu de sa phase scripturale, des pouvoirs poḯtiques - autrement dit, dès lors qu'on la considère capable d'engendrer des représentations - il est possible d'admettre que la traduction inscrit le classique dans la mémoire en même temps qu'il en écrit l'histoire. 


\section{NOTES}

1 Se manifestant « au-delà de l'interprétation sémantique des énoncés linguistiques ", lorsque "l'interprétation tend [...] à se prolonger en commentaire, dont l'ambition et la pertinence peuvent être d'ordre philosophique, esthétique et littéraire».

2 Consistant en l'application, de la part du traducteur, de catégories interprétatives formelles (d'ordre linguistique ou discursif) ou thématiques (de l'ordre de valeurs et des représentations) qui exercent une médiation entre la langue-culture de départ et la langue-culture d'arrivée.

3 Pour une étude appliquée de l'herméneutique de la temporalité dans la traduction des classiques, nous renvoyons plus particulièrement à nos analyses ciblées de la traduction de Phèdre réalisée par Ungaretti (Maggi 2020) et de la version de Dom Juan proposée par Sanguineti (Maggi 2021).

\section{BIBLIOGRAPHIE}

Aaltonen, Sirkku. 1993. «Rewriting the Exotic: The Manipulation of Otherness in Translated Drama.» In Proceedings of XIII FIT World Congress, dir. Catriona Picken, 26-33. Londres : Institute of Translation and Interpreting.

Bassnett, Susan et Peter Bush, dir. 2006. The Translator as Writer. Londres et New York, NY : Continuum. Berman, Antoine. 1984. L'épreuve de l'étranger : culture et traduction dans l' Allemagne romantique: Herder, Goethe, Schlegel, Novalis, Humboldt, Schleiermacher, Hölderlin. Paris : Gallimard.

Berman, Antoine. 1995. Pour une critique des traductions : John Donne. Paris : Gallimard.

Berman, Antoine. 1999. La traduction et la lettre ou l'auberge du lointain. Paris : Seuil.

Calvino, Italo. 1991. Perché leggere i classici. Milan : Mondadori.

Canfora, Luciano. 2000. Prima lezione di storia greca. Bari : Laterza.

Certeau, Michel de. 1975. L'écriture de l'histoire. Paris : Gallimard.

Crisafulli, Edoardo. 1999. "Tre tesi sulla modernizzazione del linguaggio della traduzione ». Testo a Fronte ottobre, $21: 113-127$.

Deniau, Guy. 2002. « Histoire et incarnation. Introduction à la question du 'sujet' chez Gadamer. » Les études philosophiques 62, $3: 333-352$.

Dionigi, Ivano, dir. 2002. Di fronte ai classici: A colloquio con i greci e i latini. Milan : BUR.

Eliot, Thomas Stearn. 1945. What is a classic ? Londres : Faber \& Faber.

Gadamer, Hans-Georg. [1960] 1996. Vérité et méthode. Traduit par Pierre Fruchon, Jean Grondin et Gilbert Merlio. Paris : Seuil.

Gadamer, Hans-Georg. 1999a. La philosophie herméneutique. Traduit par Jean Grondin. Paris : PUF.

Gadamer, Hans-Georg. 1999b. Herméneutique et philosophie. Traduit par Jean Greisch. Paris: Beauchesne.

Grondin, Jean. 2004. "L'herméneutique, de Heidegger à Gadamer. » In Le souci du passage : Mélanges offert à Jean Greisch, dir. Philippe Capelle et al., 41-60. Paris : Cerf.

Goldhill, Simon. 2010. "Cultural History and Aesthetics: Why Kant Is No Place to Start Reception Studies. » In Theorising Performance: Greek Drama, Cultural History and Critical Practice, dir. Edith Hall et Stephe Harrop, 56-70. Bristol : Bloomsbury. DOI : https://doi.org/10.5040/9781472540430. ch-005.

Hartog, François. [2003] 2015. Régimes d'historicité. Présentisme et expériences du temps. Paris : Seuil.

Holmes, James S. [1988] 1994. "The Cross-Temporal Factor in Verse Translation. " In Translated! : Papers on Literary Translation and Translation Studies, James S. Holmes, 35-44. Amsterdam et Atlanta, GA : Rodopi.

Jauss, Hans Robert. [1978] 2015. Pour une esthétique de la réception. Traduit par Claude Maillard. Paris : Gallimard.

Kermode, Frank. 1975. The Classic: Literary Image of Permanence and Change. New York, NY : The Viking Press et London : Faber \& Faber.

Ladmiral, Jean-René. 2006. "L'empire des sens. " In Le sens en traduction, dir. Marianne Lederer, 109-123. Caen : Minard. 
Laera, Margherita, dir. 2014. Theatre and Adaptation. Return, Rewrite, Repeat. Londres et Oxford : Bloomsbury.

Laera, Margherita. 2016. Translating Theatre Documentary, accédé le 22 août 2021, https://www.youtube.com/watch?v=at8SsTI9I3E.

Le Blanc, Charles. 2013. «Rhétorique et traduction à la Renaissance. La place de l'histoire en traductologie. » Des mots aux actes $5: 263-284$.

Le Blanc, Charles. 2019. Histoire naturelle de la traduction. Paris : Les Belles Lettres.

Lefere, Robin. 1994. « La traduction archaïsante : Cervantes d'après M. Molho. » Meta 39, 1 : 241-249. DOI : https://doi.org/10.7202/003454ar.

Lefevere, André. 1992. Translation, Rewriting and the Manipulation of Literary Fame. London : Routledge.

Le Goff, Jacques. 1988. Histoire et mémoire. Paris : Gallimard.

Lianeri, Alexandra et Vanda Zajko, dir. 2008. Translation and the Classic: Identity as Change in the History of Culture. Oxford : Oxford University Press. DOI : https://doi.org/10.1093/acprof:oso/9780199288076.001.0001.

Maggi, Ludovica. 2019. Herméneutique, oralité, temporalité. L'écriture traductive théâtrale de l'interprétation des classiques à la mise en voix. Phèdre et Dom Juan traduits pour la scène italienne contemporaine. Thèse de doctorat en traductologie, Université Sorbonne Nouvelle - Paris 3.

Maggi, Ludovica. 2020. " Ungaretti traducteur de 'Phèdre' : entre baroque et hermétisme, l'appropriation transhistorique d'un classique sous le signe de la déchirure. " In Tra due rive : Traduzioni letterarie d'autore nella cultura europea, dir. Francesco Laurenti, 69 -85. Canterano : Aracne editrice.

Maggi, Ludovica. 2021. « Dom Juan 'travestito'. Sanguineti traducteur et le déguisement d'un classique.» Al-Kìmiyā 19 (Janvier) : 55 -70.

Martin, Thomas. 2020. " Poiesis. » Oxford Research Encyclopediae. Literature, accédé le 22 août 2021, https://oxfordre.com/literature/browse;jsessionid=7322720287336706A8D6AD6D5F0CBC92?pageSize $=20 \&$ sort $=$ titlesort $\&$ subSite $=$ literature $\& \mathrm{t}=$ ORE_LIT\%3AREFLIT004\&t0=ORE_LIT\%3AREFLIT003.

Martindale, Charles. 1993. Redeeming the Text: Latin Poetry and the Hermeneutics of Reception. Cambridge : Cambridge University Press.

Martindale, Charles. 2013. "Reception - A New Humanism? Receptivity, Pedagogy, the Transhistorical. » Classical Receptions Journal 5, 2 : 169-183. DOI : https://doi.org/10.1093/crj/cls003.

Meschonnic, Henri. 1982a. Critique du rythme. Paris : Verdier.

Meschonnic, Henri. 1982b. «Qu'entendez-vous par oralité ? " Langue française 56 : 6-23.

Mieli, Paolo. 2002. «I classici a(l) fronte della modernità. » In Di fronte ai classici: A colloquio con i greci e i latini, dir. Ivano Dionigi, 163-174. Milan : BUR.

Monteforte, Virginia. 2019. « Moral Balance, Reciprocity and the Politics of Memory in Post-colonial Malta. » Journal of Maltese History 6, $1: 1-18$.

Nouss, Alexis. 2007. "Perspectives transhistoricistes. " TTR 20, 1 : 141-170. DOI : https://doi. org/10.7202/018501ar.

Nouss, Alexis. 2008. « Du transhistoricisme traductionnel. » In Beyond Descriptive Translation Studies : Investigations in Homage to Gideon Toury, dir. Anthony Pym, Miriam Shlesinger et Daniel Simeoni, 381-398. Amsterdam : John Benjamins. DOI : https://doi.org/10.1075/btl.75.28nou.

Nouss, Alexis. 2009. "La relation transhistorique. " In Übersetzung und Hermeneutik - Traduction et herméneutique, dir. Larisa Cercel, 293-316. Bucharest : Zeta Books.

Offenstadt, Nicolas. 2011. L'historiographie. Paris : PUF.

Paloposki, Outi. 2011. "Domestication and Foreignization. » In Handbook of Translation Studies, Volume 2, dir. Yves Gambier et Luc van Doorslaer, 40-42. Amsterdam : John Benjamins. DOI : https:// doi.org/10.1075/hts.2.dom1.

Pickering, Michael. 1999. « History as Horizon : Gadamer, Tradition and Critique. » Rethinking History 3, $2:$ 177-195. DOI : https://doi.org/10.1080/13642529908596342.

Piletić, Milana. 1999. «Il problema della distanza temporale nella traduzione delle opere letterarie. » Rivista internazionale di tecnica della traduzione 4 : 81-104.

Racine, Jean. 1984. Fedra. Traduit par Giovanni Raboni. Milan : BUR. 
Racine, Jean. 1993. Fedra. Traduit par Roberto Carifi. Milan : Feltrinelli.

Racine, Jean. 2011. Fedra. Traduit par Luigi Nazzareno Todarello. Novi Ligure : LTE.

Rega, Lorenza. 2001. «Lontananza spazio-temporale. » In La traduzione letteraria : Aspetti e problemi, Rega, Lorenza, 61-89. Torino : UTET.

Ricœur, Paul. 1985. Temps et récit. Volume 3. Paris : Seuil.

Ricœur, Paul. 2000. La mémoire, l'histoire, l'oubli. Paris : Seuil.

Roux-Faucard, Geneviève. 2001. Traduction et retraduction du texte littéraire narratif : Les métamorphoses de Kafka et de quelques autres. Thèse de doctorat en traductologie, Université Sorbonne Nouvelle - Paris 3.

Sainte-Beuve, Charles. 1850. Causeries du lundi. Paris : Garnier.

Santagata, Marco. 2002. «L'Isola Che Non Cè. »In Di fronte ai classici: A colloquio con i greci e i latini, dir. Ivano Dionigi, 215-228. Milan : BUR.

Schleiermacher, Friedrich. [1813] 1963. « Über die verschiedenen Methoden des Übersetzens. » In Das Problem des Übersetzens, dir. Hans Joachim Störig, 38-69. Stuttgart : Wissenschaftliche Buchgesellschaf.

Seleskovitch, Danica et Marianne Lederer. 1984. Interpréter pour traduire. Paris : Didier Erudition.

Settis, Salvatore. 2004. Futuro del classico. Torino : Einaudi.

Steiner, George. 1975. After Babel : Aspects of Language and Translation. New York, NY et Londres : Oxford University Press.

Stolze, Radegundis. 1992. Hermeneutisches Übersetzen. Tübingen : Gunter Narr Verlag.

Todarello, Luigi Nazareno. 2011. Communication personnelle.

Toury, Gideon. 1980. In Search of a Theory of Translation. Tel Aviv: The Porter Institute for Poetics and Semiotics.

Ungaretti, Giuseppe. 1950. Vita d'un uomo VII. Fedra di Jean Racine. Milan : Mondadori.

Vegetti, Mario. 2002. «Classico o antico?» In Di fronte ai classici: A colloquio con i greci e i latini, dir. Ivano Dionigi, 265-278. Milan : BUR.

Venuti, Lawrence. 1995. The Translator's Invisibility. Londres et New York, NY : Routledge.

Venuti, Lawrence. 1998. The Scandals of Translation: Towards an Ethics of Difference. Londres et New York, NY : Routledge.

Venuti, Lawrence. 2013. Translation Changes Everything. Londres et New York, NY : Routledge.

Wilhelm, Jane Elisabeth. 2006. "Le dialogue herméneutique. "Hermēneus. Revista de Traducción e Interpretación 8 : 155-180. 
Translation of classics. Classical reception. Hermeneutics. Temporality. Historiography. Representation.

This article aims at establishing a theoretical framework for the translation of classics. Based on hermeneutics, it presents translation as a historiographical undertaking in which the present acts as the horizon in the interpretation of the past. More specifically, translation is envisaged as an act of categorization of the work of the past within the contemporary collective imagination, according to a specific experience of temporality and a given vision of the classic as a cultural object of the past. Translative writing adds to this construction with the function of a poietic instrument, capable of generating a contemporary representation of the classic while writing its history and inscribing it in memory.

\author{
Ludovica Maggi \\ Maître de conférences - Traduction, traductologie, interprétation \\ ESIT - École Supérieure d'Interprètes et de Traducteurs \\ Université Sorbonne Nouvelle \\ Centre Universitaire Dauphine \\ 75775 Paris Cedex 16 \\ France \\ ludovica.maggi@sorbonne-nouvelle.fr \\ Chercheur associé - Communication interculturelle et traduction \\ ISIT - École de Management et de Communication Interculturels \\ 39 bis, rue d'Assas \\ 75006 Paris \\ France \\ l.maggi@isit-paris.fr
}

